\title{
THE GENETICAL RESPONSE TO NATURAL SELECTION BY VARIED ENVIRONMENTS \\ II. OBSERVATIONS ON REPLICATE POPULATIONS IN SPATIALLY VARIED LABORATORY ENVIRONMENTS
}

\author{
C. S. HALEY AND A. J. BIRLEY \\ Department of Genetics, University of Birmingham, P.O. Box 363, Birmingham B15 2TT, \\ England
}

Received 18.iv.83

\section{SUMMARY}

From each of two populations of Drosophila melanogaster, collected two months previously, from Chateau Tahbilk, S. Australia and Groningen, The Netherlands, duplicate populations were initiated in each of four environments which differed in their degree of environmental heterogeneity. Differing combinations of three food media based on oatmeal/treacle, potato or fig were used to simulate levels of environmental heterogeneity within the populations. The polymorphic loci, Adh, Est-6, G-6pdh, $\alpha$-Gpdh, Pgm, Lap-D and Aph in both the Chateau Tahbilk and Groningen derived populations and $6 \mathrm{Pgdh}$, which was only polymorphic in the populations which came from Chateau Tahbilk, were monitored in the experiment. The populations maintained a size of about 2500 adults and were sampled after 16 and 32 generations.

Large changes of phenotype frequency were shown by all loci. Despite a frequent divergence of phenotype frequencies between duplicate cages, systematic effects of occasion and environment were present and allele frequencies at many loci were shown to be changing at a faster rate than could be due to random genetic drift.

Genetic heterozygosity differed between environments but was not positively correlated with degree of environmental heterogeneity.

\section{INTRODUCTION}

An approach to the assessment of the effects of selection by different or varied environments upon enzyme and other protein polymorphisms is to study changes in genotype frequency in laboratory populations. This approach has been used to study the effects of different types of environment and also to obtain an estimate of the fraction of loci showing a selective response to the imposed environmental regimes. Examples of this method are provided by Powell (1971), McDonald and Ayala, (1974) and Minawa and Birley $(1975,1978)$. Although such studies do not conclusively demonstrate that the loci under observation are themselves the object of selection, they still exemplify the short-term response to selection and may be indicators of the extent of selection by variation in the natural environment.

An additional advantage of the study of laboratory populations in artificial or modelled environments is that it permits the examination of the maintenance of polymorphisms by, for example, either spatial or temporal variation of the environment. Theoretical models of spatial variation in which individuals mate at random and traverse the whole range of environments show that conditions for gene frequency equilibrium are very limited (Gillespie, 1974, Templeton and Rothman, 1974; Strobeck, 1975). 
The conditions become slightly less restrictive if individuals remain for a significant part of their lifespan in one environmental niche but mating is at random over all niches (Levene, 1953; Levins and MacArthur, 1966; Maynard Smith, 1966). If individuals both mate and produce progeny in a particular niche, the conditions under which genetic variation can be maintained become much less stringent (Levins and MacArthur, 1966, Levins, 1968).

Cyclical variation in the environment is also capable of maintaining genetical variation (Dempster, 1955; Haldane and Jayakar, 1963). Recently, studies of the effects of random fluctuations in the environment over time with particular reference to enzyme polymorphisms have shown that these may maintain genetical variation (Gillespie and Langley, 1974; Gillespie, 1976).

Previous studies with laboratory populations subject to environmental variation have provided evidence for a positive association between environmental heterogeneity and genetic heterozygosity. Powell (1971) has shown that the rate of loss of heterozygosity at a number of allozyme loci in Drosophila willistoni is retarded in more variable environments. A similar study was reported by McDonald and Ayala (1974) with Drosophila pseudoobscura.

In the present study a set of environments was created with a gradient of spatial environmental heterogeneity such that the more heterogeneous environments contained discrete environmental niches. Spatial environmental variation was produced by way of differing food media, the only temporal variation present in the design were aging effects of the food media, population density variation and random environmental effects. Thus the design provided a test of the effect of environmental heterogeneity upon genic heterozygosity and additionally a test for effects of environmental niches upon the enzyme polymorphism. Furthermore, two different recently collected natural populations were studied in the same set of environments in order to test for any generality of response, and the replication of large populations in all major environments allowed a comprehensive assessment of results.

\section{MATERIALS AND METHODS}

(i) The sampled populations

Two populations of Drosophila melanogaster were used in this experiment. They were a) the "Groningen" population, collected in a fruit market in The Netherlands by Dr W. Van Delden and b) the "Chateau Tahbilk" population, collected from a vineyard in Southern Australia by $\mathrm{Dr}$ A. MacKenzie. Both populations were collected as circa 100 isofemale lines and were maintained for three generations in the laboratory before the experiment commenced.

\section{(ii) The experimental populations}

For both the "Groningen" and "Chateau Tahbilk" populations four hundred virgin flies (equal numbers of both sexes) were collected from each isofemale line and placed into a population cage. The flies were allowed to intermingle for 4 hours and then the populations were each divided to 
derive to sub-populations from each of the "Groningen" and "Chateau Tahbilk" populations. Twenty vials each containing a standard oatmeal/molassess medium (see below) were placed in every cage and the populations left for 18 hours at $25^{\circ} \mathrm{C}$. After this period all of the vials were removed, discarded and replaced with fresh vials containing the same food medium. By this time most of the female flies are gravid and hence potential contributors to the next generation. After a further 24 hours at $25^{\circ} \mathrm{C}$ the vials were removed from the 'cages and the food medium in the vials was removed and quartered. Each quarter was then placed into a $\frac{1}{3}$ pint milk bottle again containing the standard oatmeal/molasses (see later) food medium. Each bottle received about 300 eggs and the food medium was sufficient to support at least 500 larvae, without intense competition, at $25^{\circ} \mathrm{C}$. The emergent adults were collected from all of the bottles and placed in a population cage. After intermingling for 4 hours the flies were removed and 2000 flies, equal numbers of both sexes, were placed into the experimental cages. All cages were maintained at $25^{\circ} \mathrm{C}$ and originally contained 18 tubes of standard oatmeal/molasses food medium.

\section{(iii) The experimental environments}

Three types of food medium were used in different combinations with each other. They were standard oatmeal/molasses medium (type A), fig based (type B) and potato based (type C). Type A medium consisted of $72 \mathrm{~g}$ oatmeal (Mornflake, fully stabilized); $35 \mathrm{~g}$ molasses (Fowler's); $6 \mathrm{ml}$ Nipagin-M as a 1 per cent solution in 95 per cent ethanol; 6 gms agar (Köbe) and $400 \mathrm{mls}$ water. Type B was $100 \mathrm{~g}$ dried figs subsequently rehydrated and homogenised in $500 \mathrm{ml}$ water; $15 \mathrm{~g}$ dried flaked, killed baker's yeast; $5 \mathrm{~g}$ agar (Köbe) and $5 \mathrm{mls}$ Nipagin solution. Type C comprised $50 \mathrm{~g}$ dried potato; $500 \mathrm{ml}$ water; $15 \mathrm{~g}$ dried flaked yeast; $5 \mathrm{~g}$ agar (Köbe) and $5 \mathrm{ml}$ Nipagin solution. Each vial of food medium contained $8 \mathrm{ml}$ of one of these media, medium type A was additionally topped with $1 \mathrm{ml}$ of live baker's yeast suspension. Four types of environment were established from both the "Groningen" and "Chateau Tahbilk" derived populations. the environments can be represented by three letters each representing one of the three vials of food medium, with which the populations were supplied for their maintenance. The four environments were: (1) AAA; (2) AAB; (3) AAC; (4) ABC. Hence the ABC environments were supplied with one vial of each of the three types of medium on each feeding occasion. Duplicate cages of all environments were established there being 16 cage populations in all. In addition, a series of populations was also established with the environmental compositions, $\mathrm{ABB}, \mathrm{ACC}, \mathrm{BBB}, \mathrm{BBC}, \mathrm{BCC}$ and $\mathrm{CCC}$. For reasons of space, this series was unreplicated and only set up with flies from the "Groningen" population.

The cages were maintained at $25^{\circ} \mathrm{C}$. The cycle of media vial changes was over two weeks, three vials being changed on Monday, Wednesday and Friday of the first week and Tuesday and Thursday of the second week. Each cage contained 18 vials of medium thus the mean time a vial spent in a cage was 16.8 days. By this time all flies had emerged and the food medium had been fully utilised.

The experimental size necessitated the use of two identical incubators. In an attempt to compensate for the effect of any slight difference between 
the incubators, the population cages were randomly re-allocated to the various positions within the incubators every time new food was supplied. This procedure has the effect of distributing over cages any environmental variance between different incubators and shelves within incubators.

\section{(iv) Sampling the environments}

All populations were sampled (see below) 11 and 22 months after their establishment. Allowing a generation interval of 3 weeks for Drosophila melanogaster populations with overlapping generations (Barker, 1962), these periods represent generations 16 and 32 respectively and will be referred to as sampling occasions one and two. Additionally, all environments containing only one type of medium or all three types of media were sampled two months, i.e., three generations, after the initiation of the experiment, this will be referred to as sampling occasion zero.

Three sub-samples of eggs were taken from every cage when it was sampled, hence all the component types of medium were sampled from each cage. The minimum number of individuals typed from each locus from each of the three sub-samples within a population cage on each occasion was approximately 30 . There was no more variation between sub-samples within cages than would be expected due to chance, thus the sub-samples within a population cage were pooled for the purposes of analysis. The egg samples were raised at low density at $250^{\circ} \mathrm{C}$ in culture bottles containing medium of the same type as that upon which the eggs were sampled. Third instar larvae were collected for typing with respect to the Adh locus and late pupae for typing at the Lap-D locus. Emergent flies were aged for 4 days after collection and then typed with respect to the $A d h, E s t-6, G-6 p d h$, Pgm and $\alpha-G p d h$ loci in populations. 6-Pgdh was only polymorphic and hence typed in the series of populations derived from "Chateau Tahbilk". After collection all samples were stored at $-20^{\circ} \mathrm{C}$ prior to gel electrophoresis.

\section{(v) Gel electrophoresis}

Gel-electrophoresis of individual fly homogenates was carried out in $\mathbf{1 2}$ per cent starch gels (Connaught Medical Laboratories, Toronto). Poulik's (1957) discontinuous buffer system was used to type Lap-D, Adh, Est-6 and $\alpha-G p d h$. A Tris-citrate continuous buffer system $p H$ 7.0 (Ayala, 1972), was used to type G6Pdh, Pgm and 6Pgdh and a tris-broate-EDTA continuous buffer was used for $A d h$ (Wallis and Fox, 1968).

\section{(vi) Data}

All polymorphic loci studied had two electromorphs apart from the Est-6 and Pgm loci in the "Chateau Tahbilk" population which both had two additional rare electromorphs. All electromorphs present in the "Groningen" population were also present in the "Chateau Tahbilk" population. The amount of data collected precludes its full inclusion here. However, a summary of the data, in the form of the frequency of one allele class from each locus in each population cage on each sampling occasion, is given in table 1 for the "Chateau Tahbilk" populations and in table 2 
for the "Groningen" populations. The allele classes shown in Tables 1 and 2 are those which were commonest in the "Chateau Tahbilk" populations.

\section{Results}

\section{(a) Phenotypic frequencies}

The variation in phenotypic frequencies at a locus was analysed in respect of environments, sampling occasions and replicate populations using the log-likelihood ratio statistic $G^{2}$ or $2 I$ (Kullback, 1959). The analysis permits the total homogeneity of phenotypic frequency at a locus to be partitioned in accordance with table 3. The partitions of the total homogeneity of phenotype frequencies of any one locus represent:

(1) homogeneity of phenotypes over environments, irrespective of sampling occasion and replicate population (environments).

(2) homogeneity of phenotypes over occasions, irrespective of environments (occasions).

(3) the homogeneity of phenotypes over environments conditional upon occasions (environments $X$ occasions).

(4) the homogeneity of phenotypes over replicates within environments (replicates within environments).

(5) the homogeneity of replicates within environments conditional upon occasions (replicates within environments $\times$ occasions).

The appropriate analyses for "Chateau Tahbilk" populations are shown in table 4 and for "Groningen" populations, ir Table 5. All loci, except Pgm and Est-6 in "Chateau Tahbilk" were di-allelic (i.e., had two allele mobility classes) and thus had three phenotypic classes. However, at the Adh, G-6Pdh and Pgm loci in the "Groningen" population one homozygote was quite rare and was pooled with the heterozygote for the purposes of analysis in order to avoid having classes with expected numbers of less than 4.

Pgm and Est-6 are tetra-allelic in the "Chateau Tahbilk" population with allele frequencies such as to give expected numbers some classes of phenotype less than 4 . Consequently the rarest phenotypic classes corresponding to these loci were pooled to give classes with expected values greater than 4. This pooling resulted in three phenotypic classes for $\mathrm{Pgm}$ and four phenotypic classes for Est-6.

The $G^{2}$ or $2 I$ values from each of the five items in this analysis are tested for significance by comparison with the $x^{2}$-distribution for the appropriate degrees of freedom. The analyses show a substantial change in phenotype frequency due to divergence between replicate populations. This is shown both in the item testing homogeneity between replicate populations within environments (item 4 ), and the item testing the homogeneity of replicate populations within environments and occasions (item 5), which are often significant at least the 5 per cent level. A significant $G^{2}$ value for one of the main effects, that is the environments item (item 1), the occasions item (item 2) on the environments $\times$ occasions item (item 3), can only be interpreted as demonstrating an effect in the absence of a significant $G^{2}$ value for the appropriate error item. Thus the effect of environments (item 1) was assessed relative to the variation between replicate cages within environments (item 4) and the effects of occasions (item 2) and 


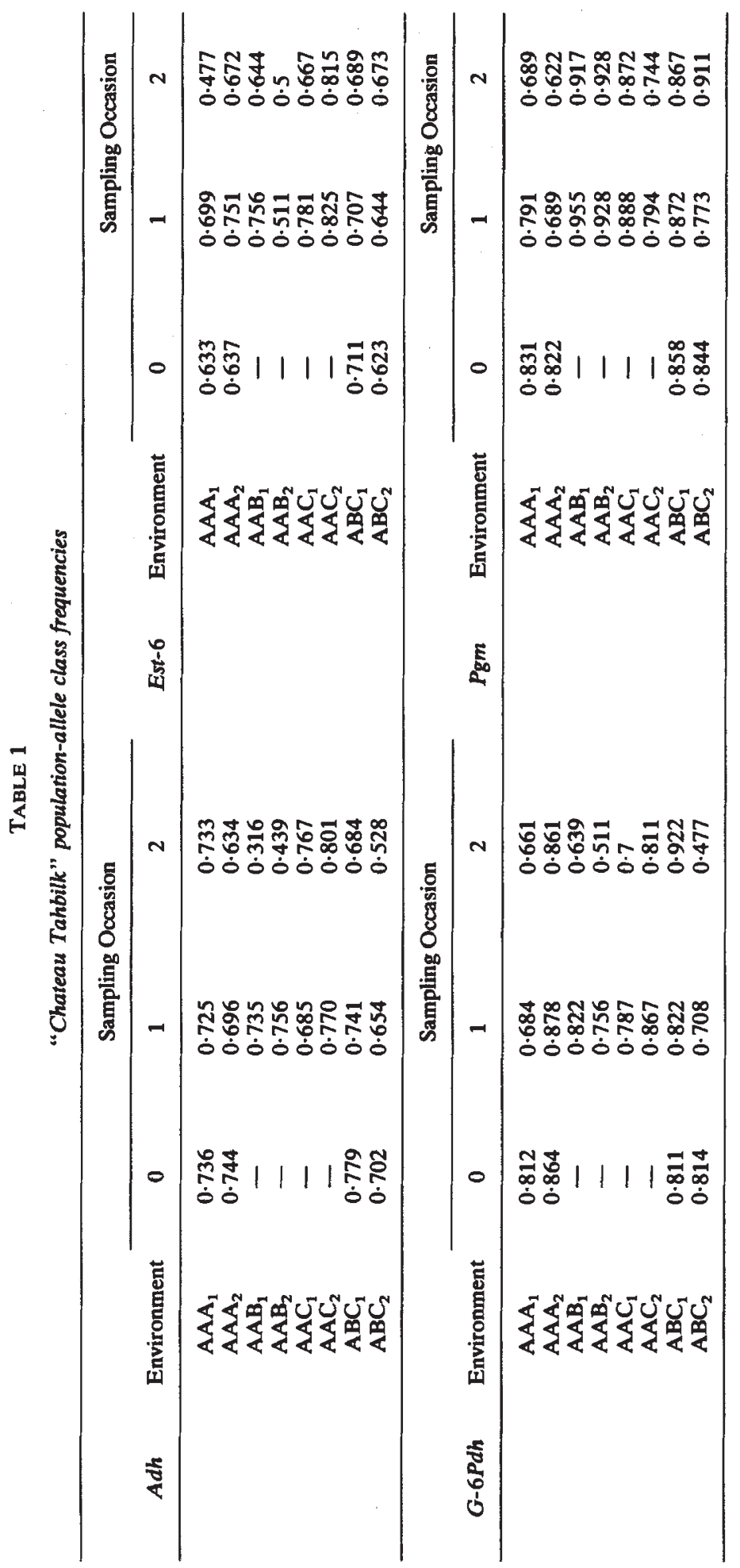




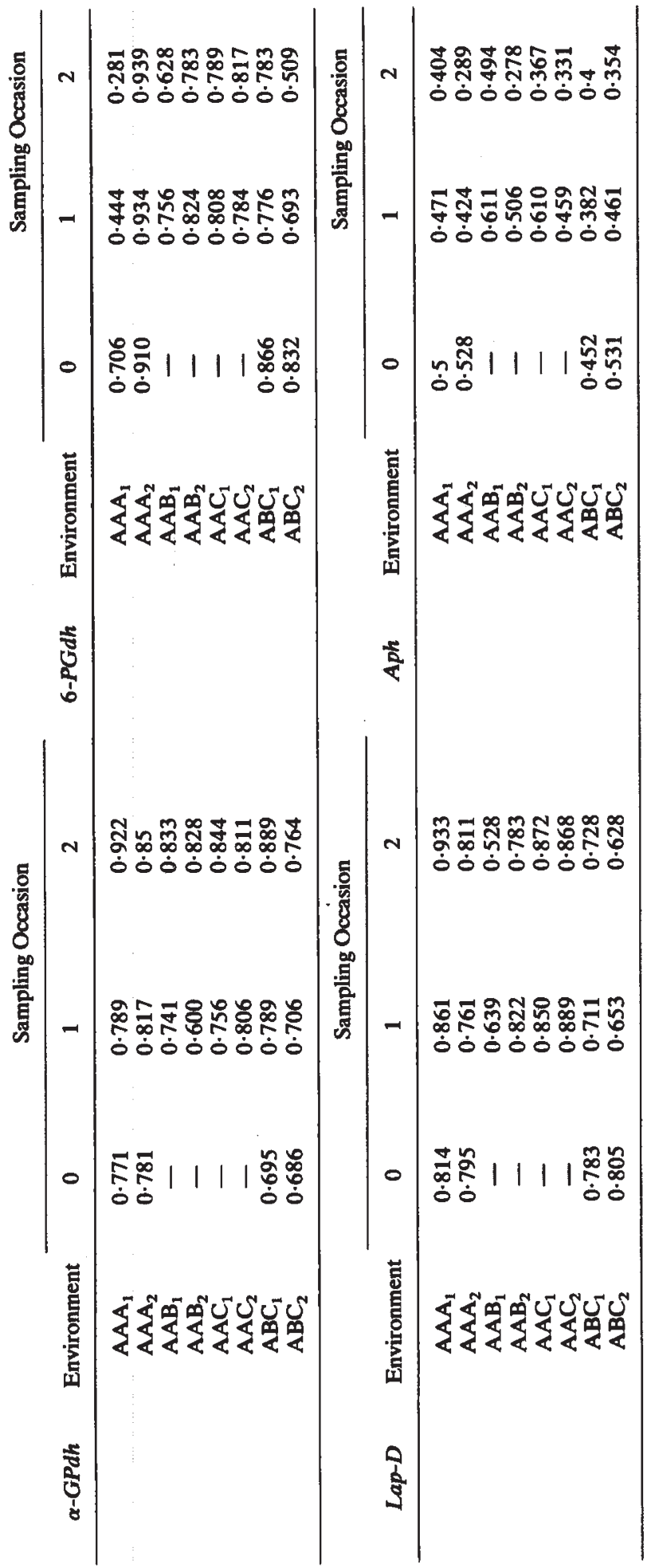




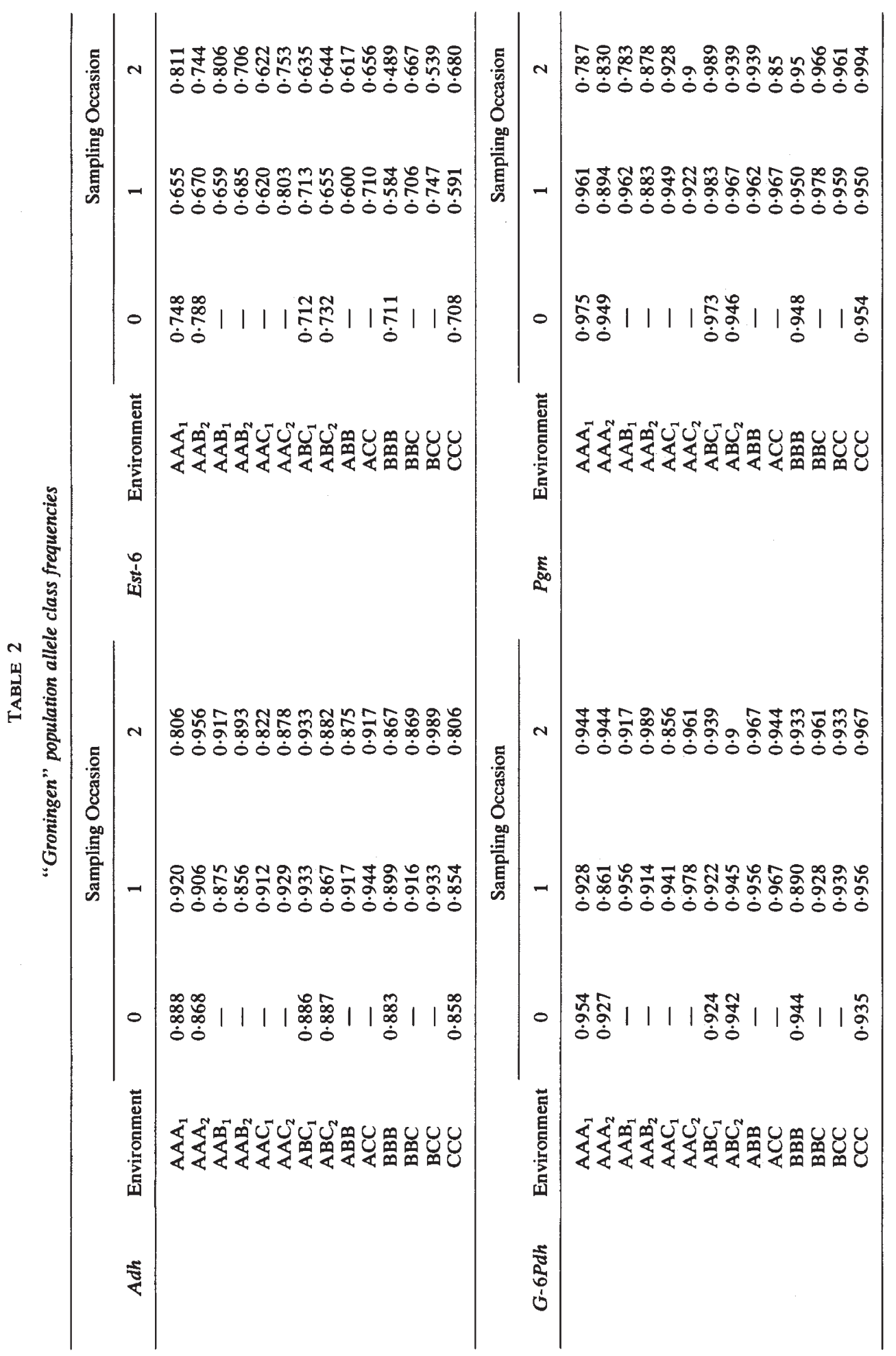




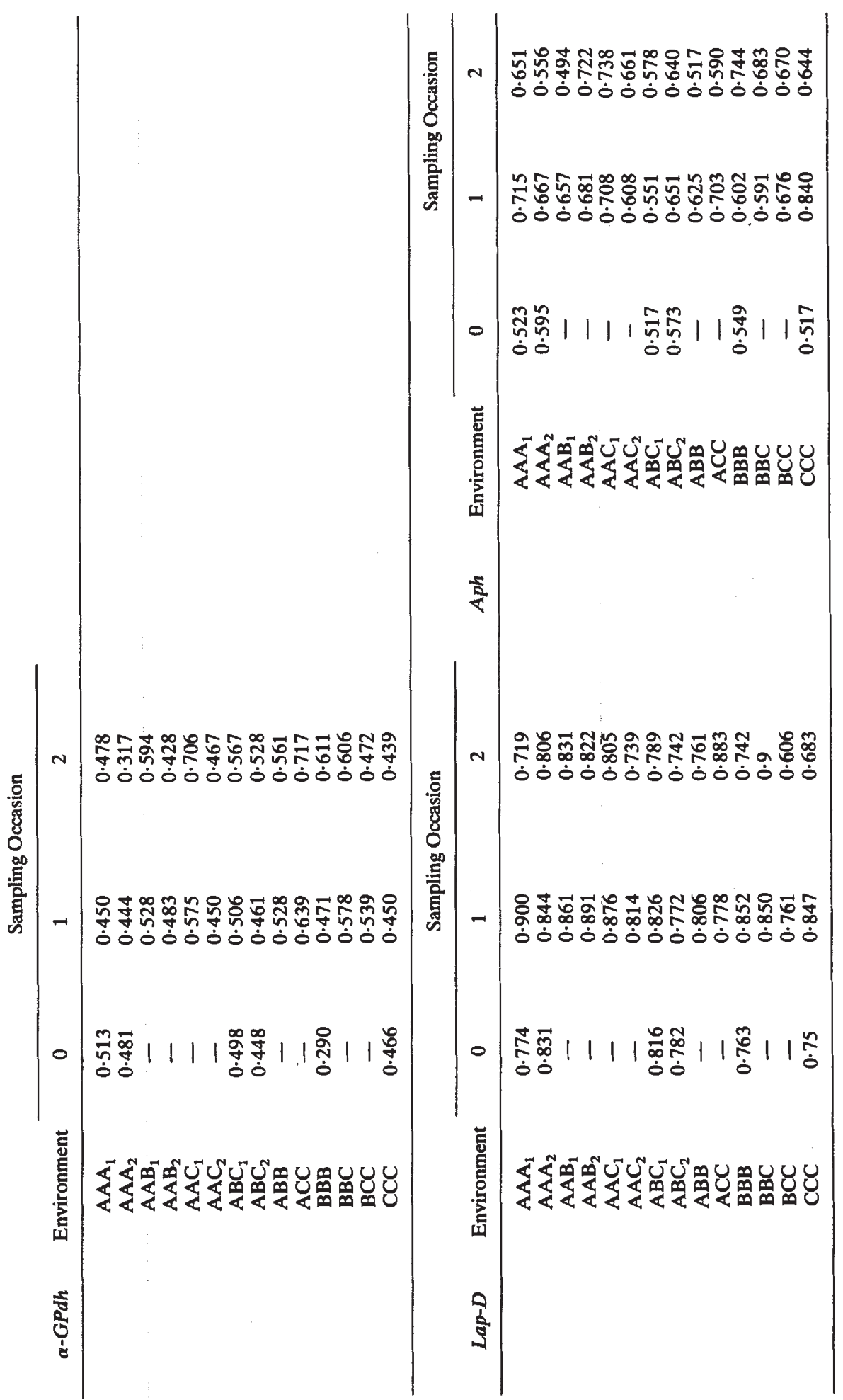




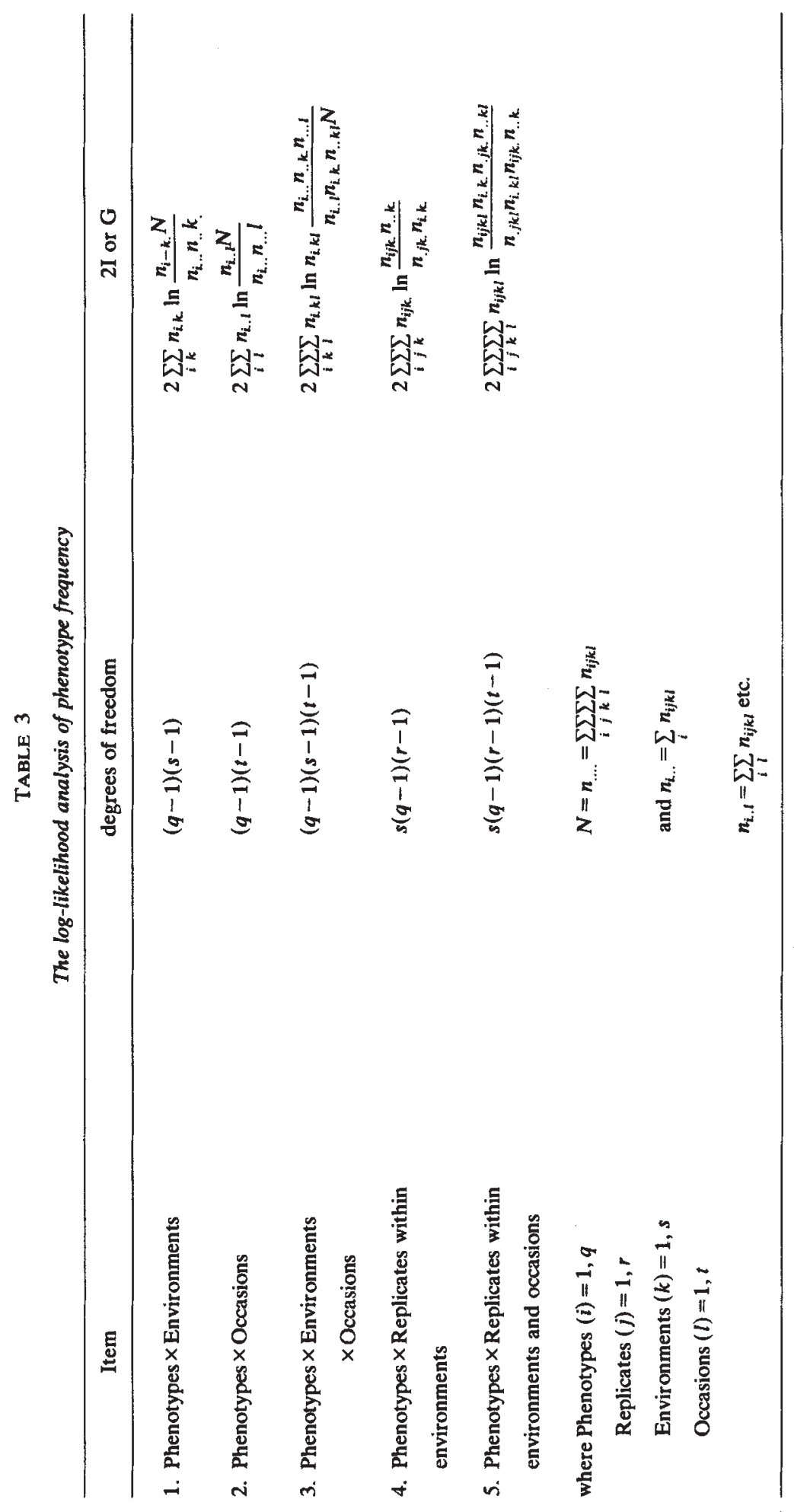


environments $\times$ occasions (item 3 ) were assessed relative to item 5 , replicate cages within environments $\times$ occasions.

The only cases in which the main effects are significant and the appropriate error terms are not significant are: in the "Chateau Tahbilk" populations; the occasions and environments $\times$ occasions items for the Adh locus and the occasions items for the Aph locus; and, in the "Groningen" populations; the occasions and environments $\times$ occasions items for the Est- 6 locus, the environments item for the Pgm locus and the occasions item for the Lap-D locus. As an approximate, and probably conservative, test for those main effects which are significant as $G^{2}$ values but which had significant error terms, an $F$-ratio test following that described by Minawa and Birley (1978) was performed. The $F$-ratio is constructed from the $G^{2}$ value for the item to be tested over its degrees of freedom divided by the appropriate error term, also over its degrees of freedom. This test reveals a further two cases in which the effect of occasions is significantly greater than its appropriate error; for the G-6Pdh and $\alpha-G p d h$ loci in the "Chateau Tahbilk" population, and a further one case in which the effect of environments is significantly greater than its appropriate error; for the Adh locus in the "Chateau Tahbilk" population. Thus for every locus studied, other than 6-Pgdh, in one or other of the two populations studied, there is evidence for systematic changes in phenotype frequency indicative of the action of selection. For all loci other than 6-Pgdh and Pgm in one or other of the populations there is evidence for an effect of occasions. However, for only three loci (Adh in "Chateau Tahbilk" and Est-6 and Pgm in "Groningen") does the presence of a significant environments or environments $X$ occasions item demonstrate any differential effect of the experimental environments.

Inspection of tables 4 and 5 reveals little similarity between the "Groningen" and "Chateau Tahbilk" populations in the systematic responses of individual loci. There are no instances where a locus produces a significant occasions item in both populations, thus there is little evidence that pure directional selection acting solely on the loci under observation. Additionally, it does not seem as though the two populations are converging on common allele frequencies; inspection of tables 1 and 2 reveals that the general trend of allele class frequencies for a locus in a population for which there is a significant occasions item, is away from the prevailing allele class frequency in the other population for all six loci with a significant occasions item apart from Lap-D. Similarly, there is little concordance between the responses to the experimental environments in the two populations. The two loci which produced significant environments items were $A d h$ (in "Chateau Tahbilk") and Pgm (in "Groningen", although the $F$ ratio in "Chateau Tahbilk" is approaching significance: $p=0.056$ ) the mean common allele class frequency for each of the four replicated environments in each population on sampling occasion 2 for these loci are shown below:

\begin{tabular}{|c|c|c|c|c|c|}
\hline \multicolumn{3}{|c|}{ Adh } & \multicolumn{3}{|c|}{ Pgm } \\
\hline Environment & $\begin{array}{l}\text { Chateau } \\
\text { Tahbilk }\end{array}$ & Groningen & Environment & $\begin{array}{l}\text { Chateau } \\
\text { Tahbilk }\end{array}$ & Groningen \\
\hline AAA & 0.684 & $0 \cdot 881$ & AAA & 0.656 & 0.809 \\
\hline $\mathrm{AAB}$ & $0 \cdot 378$ & 0.905 & $\mathrm{AAB}$ & 0.923 & 0.831 \\
\hline AAC & 0.784 & 0.850 & AAC & $0 \cdot 808$ & 0.914 \\
\hline $\mathrm{ABC}$ & 0.606 & 0.908 & $\mathrm{ABC}$ & 0.889 & 0.964 \\
\hline
\end{tabular}




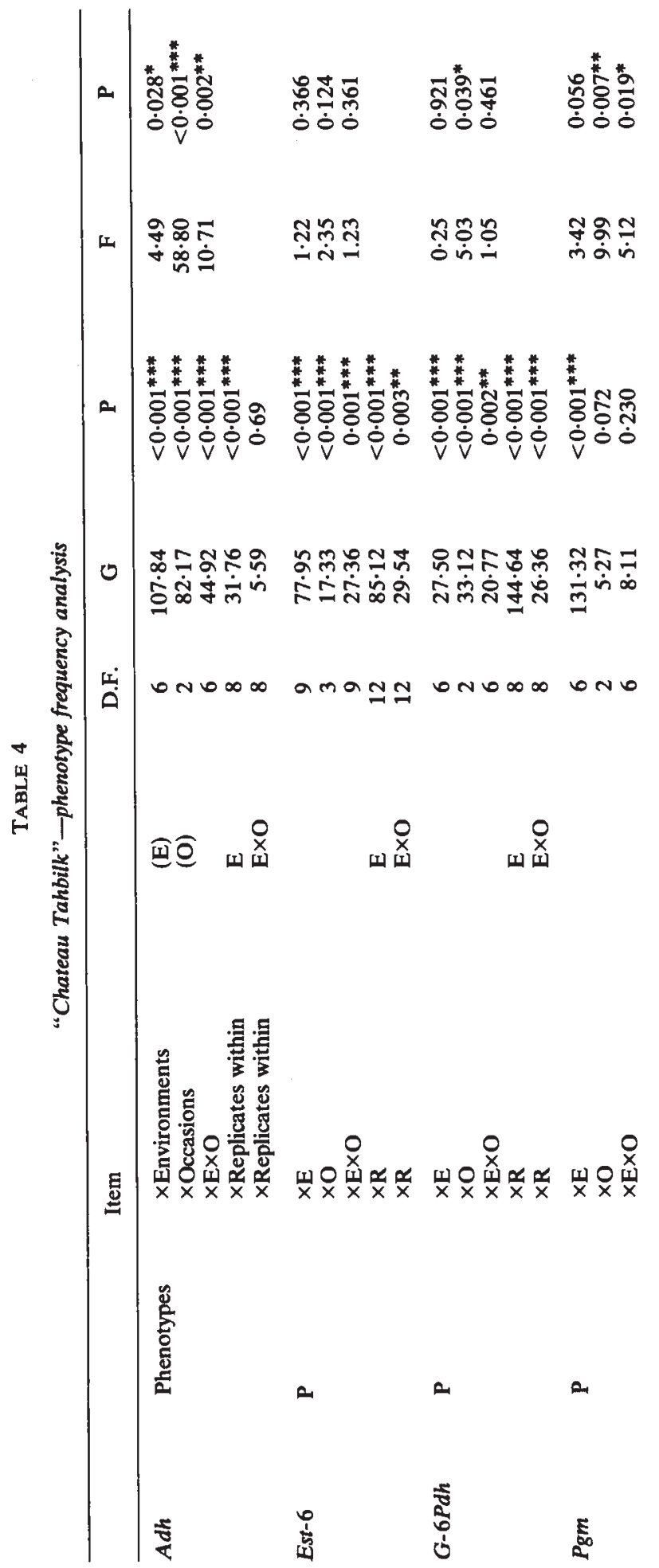




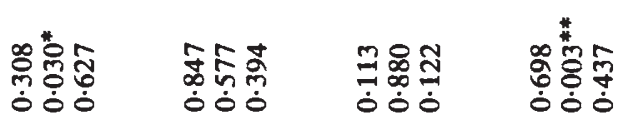

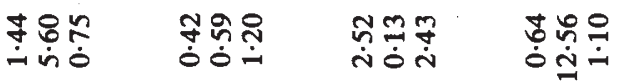

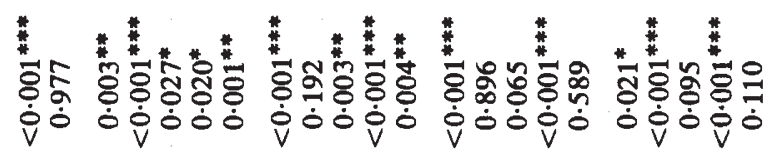

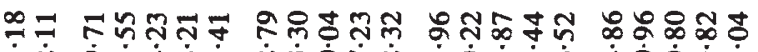

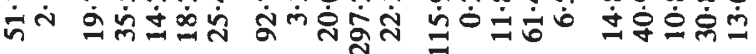

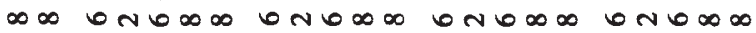

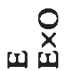
니 ํㅗ
피 비
ำ
w)

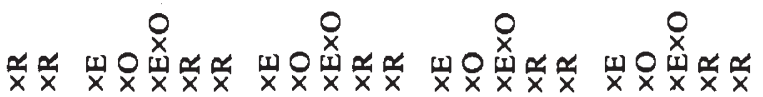
A.
a
a
a
$\frac{5}{5}$
$\frac{5}{5}$
高
客 


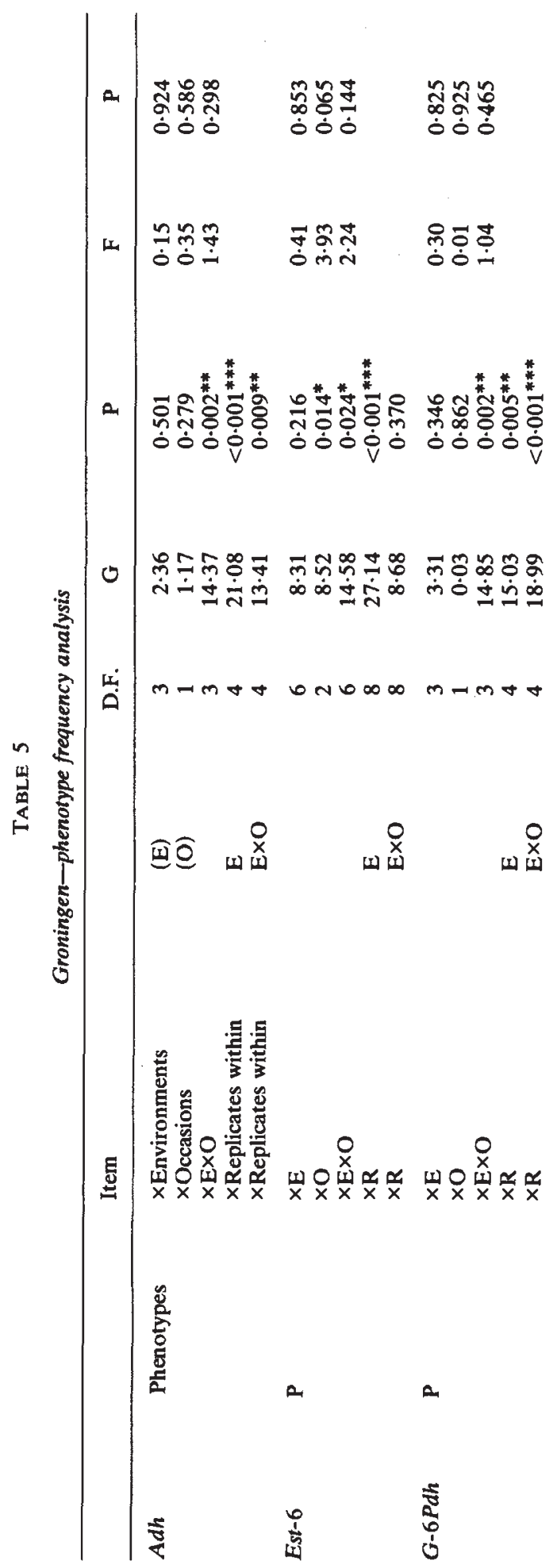




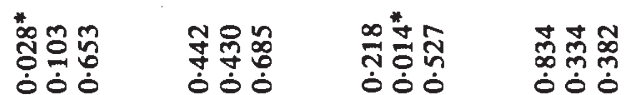

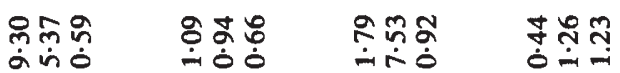

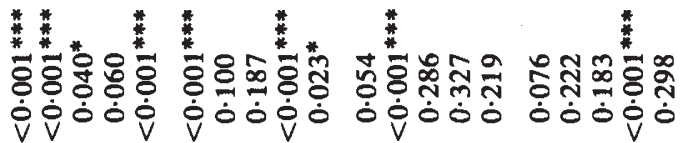

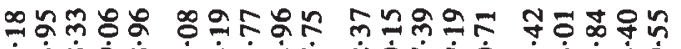

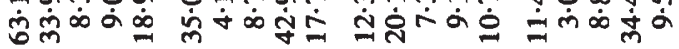

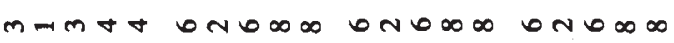

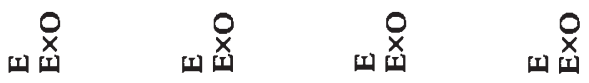

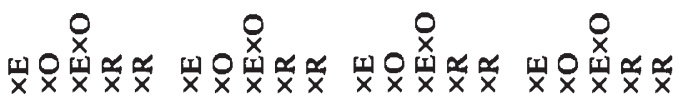

A

A

A

ค

$2^{\infty}$

宽

音

豪 
As inspection of these figures reveals, there is no similarity between the two populations for the Adh locus; the environments producing the two lowest frequencies in "Chateau Tahbilk" produce the highest ones in "Groningen". At the Pgm locus the AAA environment produces the lowest frequency in both populations but there is little similarity between the other environments.

In order to compare the overall degree of change in the two populations the average value of $G^{2}$ (i.e., the total value of $G^{2}$ over the total degrees of freedom) over all loci for each of the five items in the analysis were calculated and are shown below:

\begin{tabular}{|c|c|c|c|c|}
\hline & \multicolumn{2}{|c|}{ Chateau Tahbilk } & \multicolumn{2}{|r|}{ Groningen } \\
\hline & d.f. & Average $G^{2}$ & d.f. & Average $G^{2}$ \\
\hline Phenotypes $\times$ Environments $(\mathrm{E})$ & 51 & $11 \cdot 53$ & 33 & $4 \cdot 12$ \\
\hline X Occasions $(\mathrm{O})$ & 17 & $12 \cdot 82$ & 11 & 6.46 \\
\hline$\times \mathrm{E} \times \mathrm{O}$ & 51 & $3 \cdot 10$ & 33 & $2 \cdot 34$ \\
\hline$\times \bar{R}$ eplicates within $\mathrm{E}$ & 68 & $10 \cdot 59$ & 44 & $3 \cdot 61$ \\
\hline$\times$ Replicates within $\mathrm{E} \times \mathrm{O}$ & 68 & 1.92 & 44 & $2 \cdot 23$ \\
\hline
\end{tabular}

It can be seen from these figures that the first three averages are all larger than their errors and that this is especially so for the two occasions items. Also most items in the "Chateau Tahbilk" population are appreciably larger than in the "Groningen" population. This is despite the fact that the item measuring replicate divergence between sampling occasions 1 and 2 (phenotypes $\times$ replicates within $\mathrm{E} \times \mathrm{O}$ ) is approximately the same in both populations. The large averages for phenotypes $\times$ replicates within environments compared with those for phenotypes $\times$ replicates within environments $\times$ occasions indicates that most replicate divergence occurred prior to sampling occasion 1 .

\section{(b) Allele frequencies}

Analysis of phenotypic frequencies have demonstrated a good deal of genetic change has occurred in the set of populations. A small proportion of the response to selection can be attributed or related to the effect of environments. A substantial response to selection has, however, occurred between the two times of sampling, i.e., over 16 generations. It also appears that some of the populations may be evolving uniquely and largely independently of their environments. Therefore an analysis of the data is required which examines changes of allele frequency in replicate populations making allowance for the effects of random genetic drift. That is, we require to separate the effects of natural selection and random genetic drift as causes of the non-systematic changes seen in replicate populations. The experiment spanned approximately 32 generations and the observed population sizes (N) maintained in the cages were about 2500 to 3000 adults. In this case allele frequency changes observed between sampling occasions must be largely due to natural selection since the number of generations is much less than the effective population size ( $\mathrm{Ne}$ ) provided that $\mathrm{Ne} / \mathrm{N}$ for Drosophila melanogaster is taken to be about 0.8 (Crow and Morton, 1955). 
A test for selection taking into account the effect of random genetic drift is available from the method of Fisher and Ford (1947). These authors define the sampling variance of the observed allele frequencies around the population allele frequency as

$$
V\left(p_{i}\right)=\frac{\hat{p}_{i} \hat{q}_{i}}{2 n_{i}}-\frac{\hat{p}_{i} \hat{q}_{i}}{2 N_{i}}
$$

where $N_{i}$ is the total population size on sampling occasion $i, n_{i}$ is the sample taken on occasion $i$ and $p_{i}$ and $q_{i}$ are the allele frequencies on occasion $i$.

This relationship is for sampling from a finite population without replacement. In the present experiment the population samples were taken as samples from continuously produced egg-population rather than a population of adult flies. Hence we can omit the quantity $p_{i} q_{i} / 2 N_{i}$. Fisher and Ford (1947) demonstrated that following the angular transformation $\theta_{i}=$ $\sin ^{-1} \sqrt{p_{i}}$, the sampling variance of $\theta$ was independent of its magnitude and

$$
\begin{aligned}
V\left(\theta_{i}\right) & =\frac{180^{2}}{4 \pi^{2}} \frac{\operatorname{Var}\left(p_{i}\right)}{p_{i} q_{i}} \\
& =\frac{820 \cdot 7}{2 n_{i}} .
\end{aligned}
$$

For a chosen reference generation $(r)$ the variance of the observed allele frequency about its actual value in the reference generation is $820 \cdot 7 / 2 n_{r}$ The variance of the transformed observed allele frequency in any generation (i) about its actual value in the referenced generation due to sampling and random genetic drift then becomes $820 \cdot 7 / 2 n_{i}+x \cdot 820 \cdot 7 / 2 N$ where $x$ is the number of generations separating the two samplings. A chi-square test for selection is obtained as:

$$
\chi^{2}(j-1)=\sum_{i} T_{i} \boldsymbol{\theta}_{i}-\hat{\theta} \sum_{i} T_{i}
$$

where $j$ is the number of samples taken, $\boldsymbol{T}$ is a matrix obtained as the product of $\boldsymbol{\theta}$, a column vector of the observed values of $\theta_{i}$, and $I$ the inverse matrix of the expected variances of the estimates of $\theta_{i}$ around its population value in the reference year; these values are arrayed along the leading diagonal of the matrix. The maximum likelihood estimate $\theta$ of $\theta$ in the reference year is

$$
\hat{\theta}=\sum_{i} T_{i} / \sum_{i j} I_{i j}
$$

we of course assume random union of gametes.

Data was available from all population cages on sampling occasions 1 and 2 (approximately generations 16 and 32 respectively) and this was supplemented with data from occasion 0 (generation 3 ) where available. The Est-6 and Pgm loci in the "Chateau Tahbilk" population have four alleles but as for all other loci, only changes in the commonest allele class were included on the analysis. The results are presented in table 6 for the "Chateau Tahbilk" populations and in table 7 for the "Groningen" populations. Given $\mathrm{Ne}$ is about 2000 all loci show significant $\chi^{2}$ values. Hence the allele frequency changes are much too rapid to be accounted for by random 


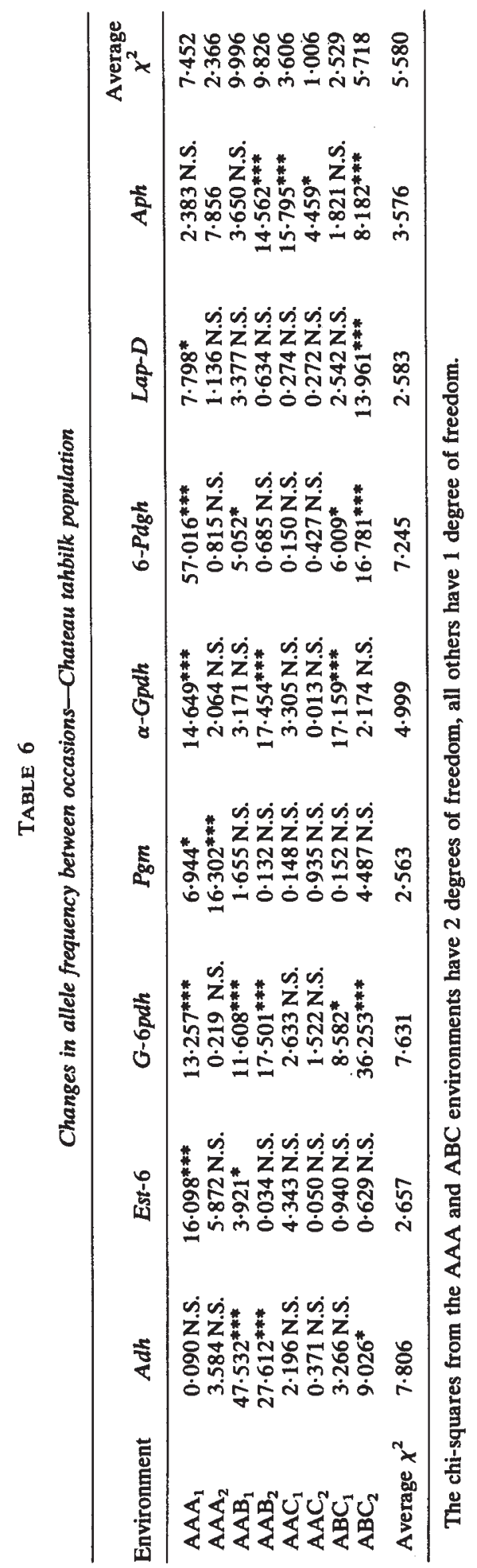




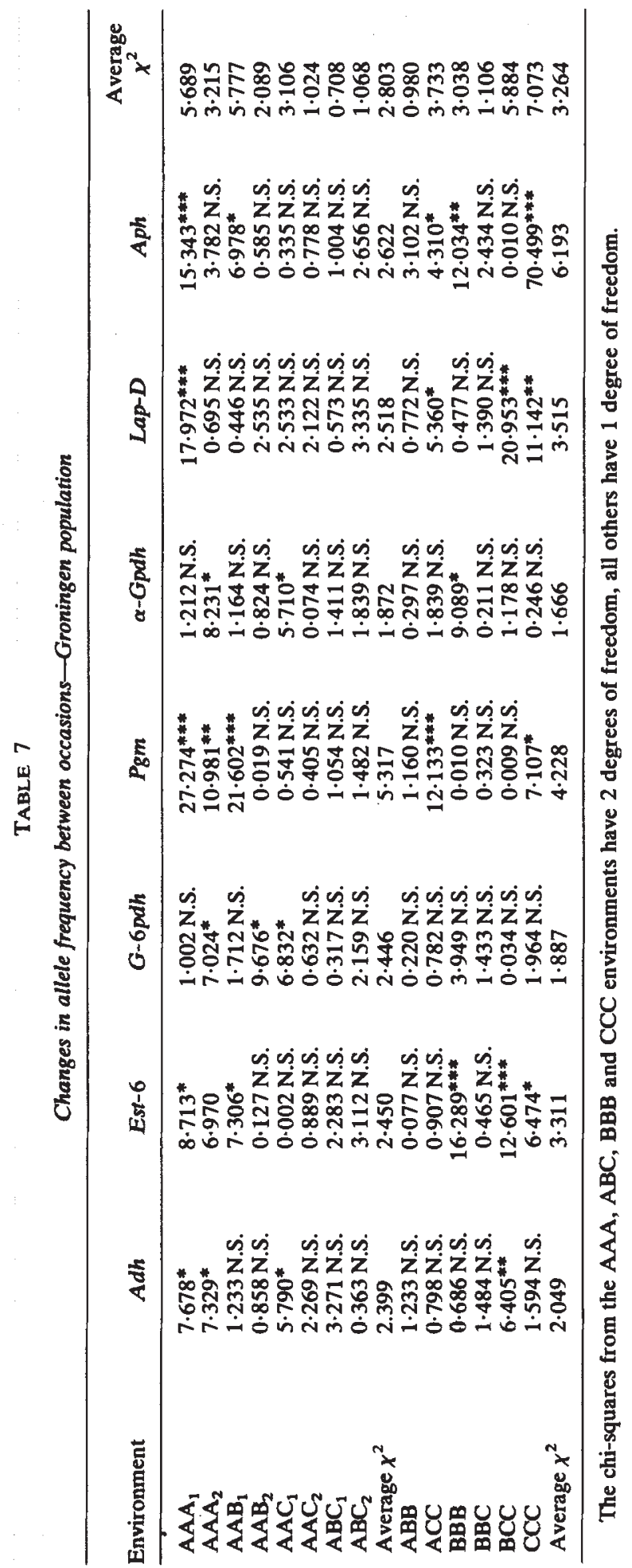


genetic drift alone. If only environments common to both sets of populations are considered, the average $\chi^{2}$ is greater in the "Chateau Tahbilk" (5.58) than in the Groningen population (2.80). Inspection of the average $\chi^{2}$ for loci shows least evidence for selection (i.e. low average $\chi^{2}$ values) at the Lap-D, Est-6 and Pgm loci in "Chateau Tahbilk" and similarly at the Adh, $G 6 p d h$ and $\alpha-G p d h$ loci in the "Groningen" populations.

As predicted from earlier results replicate populations can differ considerably in behaviour. There are cases where a replicate population shows several highly significant $\chi^{2}$ values and its counterpart none. Also allele frequencies can change in quite different directions; in the $\mathrm{ABC}$ environment of the "Chateau Tahbilk" population one replicate shows the common G-6Pdh allele to be at $0 \cdot 811,0 \cdot 822$ and 0.922 to be on the three respective sampling dates in one replicate and $0.814,0.708$ and 0.477 respectively in the duplicate population.

This example is, however, unusual, for of the 35 out of 60 cases in which there is a significant change in allele frequency in one or both duplicate populations, in 26 cases (when 3 sampling dates were included for analysis) the allele frequency changes in replicate cages were in the same direction. Hence there is a great deal of evidence to support the action of natural selection.

It might be argued that the effective size in experimental populations is substantially lower than that which we have used, and that the progeny of very few females contribute to the individual vial populations. However, in this experiment we have not detected significance between sub-samples within population cages implying that $\mathrm{Ne}$ is not small. Nonetheless we can suitably amend the analysis of data using the "Fisher-Ford" method by reducing $\mathrm{Ne}$ until the average $\chi^{2}$ in the experiment is such as to give 5 per cent of the cases statistically significant, i.e., by chance alone. This would give an effective population size of approximately 150 in "Chateau Tahbilk" and 240 in Groningen populations, giving a value of $N_{e} / N$ of about $0 \cdot 06-0 \cdot 1$ which seems highly unlikely given the size and vigour of the adult populations.

Although random genetic drift must be invoked as at least the initiator of replicate differences within environments, there is little doubt that natural selection has been a major cause of the changes in allele frequencies seen during the course of this experiment.

\section{(c) Genic heterozygosity}

The design of this experiment provides an ideal opportunity to examine the relationship between environmental heterogeneity and genic heterozygosity. If, as has been predicted by some authors, there is a positive relationship between environmental heterogeneity and genic heterozygosity, environments containing three types of food medium should be on average more heterozygous than those containing two types of medium which should in turn be more heterozygous than those containing a single type of medium. In order to examine this hypothesis the average observed heterozygosity for each level of environmental heterogeneity on each occasion was calculated and is shown in table 8 .

Inspection of Table 8 reveals no evidence for a simple positive relationship between environmental heterogeneity and genic heterozygosity. 
TABLE 8

Average observed heterozygosity for each environmental type and level of heterogeneity for the "Chateau Tahbilk and "Groningen" populations on sampling occasions 1 and 2

\begin{tabular}{llcccc}
\hline \multicolumn{2}{c}{ Level of } & \multicolumn{2}{c}{ "Chateau Tahbilk" } & \multicolumn{2}{c}{ "Groningen" } \\
Heterogeneity & Environment & Occasion 1 & Occasion 2 & Occasion 1 & Occasion 2 \\
\hline 1 type of Medium & AAA & 0.3949 & 0.3682 & 0.2951 & 0.3163 \\
2 types of Medium & AAB & 0.3541 & 0.3878 & 0.3046 & 0.2965 \\
& AAC & 0.3207 & 0.3164 & 0.2693 & 0.3159 \\
& Average & 0.3374 & 0.3521 & 0.2870 & 0.3060 \\
3 types of Medium & ABC & 0.4239 & 0.3960 & 0.3185 & 0.3212 \\
\hline
\end{tabular}

However, there is some evidence that heterozygosity varies with environmental heterogeneity as environments containing two types of food medium have the lowest average heterozygosity. Thus the metric warrants further investigation.

To facilitate analysis the average proportion of heterozygotes for every locus at every cage at each sampling occasion was transformed using the arcsine transformation. The analyses of variance of the transformed data are shown in table 9 for each combination of population ("Chateau Tahbilk" or "Groningen" derived) and sampling occasion. The variation between environments was partitioned into three orthogonal comparisons designed to locate the possible sources of any differences between the environments. The coefficients corresponding to the three comparisons $C_{1}, C_{2}$ and $C_{3}$ are defined below:

\begin{tabular}{cccc}
\hline & \multicolumn{3}{c}{ Comparison } \\
\cline { 2 - 4 } Environment & $C_{1}$ & $C_{2}$ & $C_{3}$ \\
\hline AAA & +1 & +1 & +1 \\
AAB & -1 & +1 & -1 \\
AAC & +1 & -1 & -1 \\
ABC & -1 & -1 & +1 \\
\hline
\end{tabular}

If increased environmental heterogeneity leads to an increased level of genic heterozygosity and this relationship is linear, then both $C_{1}$ and $C_{2}$ should be statistically significant and $C_{3}$ should not. If only one food medium affects the level of heterozygosity, for example, by maintaining larger population sizes and thus higher levels of heterozygosity, than either $C_{1}$ or $C_{2}$ may be signiflcant but $C_{3}$ will not. If differences in the level of heterozygosity are associated with interactions between the types of food medium then the $C_{3}$ item may reach statistical significance.

In the analysis shows in table 9 it was never the case that both $C_{1}$ and $C_{2}$ were significant, as would be the case if environmental heterogeneity per se led to increased genic heterozygosity. The loci item is highly significant in all analyses but this just indicates that there are differences in heterozygosity between loci and is here of little interest. Additionally, the replicates item is significant in all analyses when compared with the expected binomial variance; this is hardly surprising in view of the extent of replicate divergence 
TABLE 9

Analysis of variance of angular transformed, proportion heterozygosity for the "Chateau Tahbilk" and "Groningen" populations on sampling occasions 1 and 2.

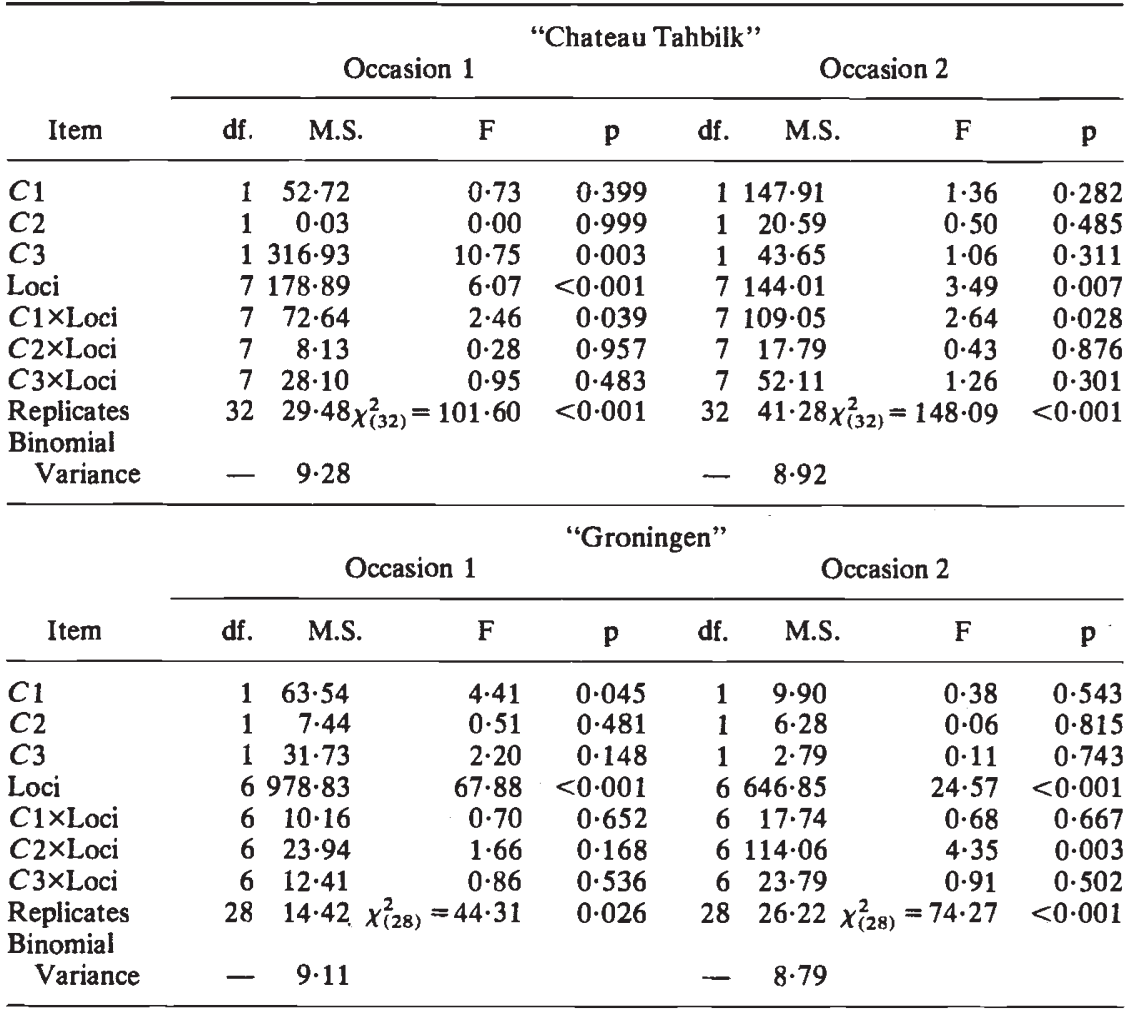

observed in previous analyses. The $C_{1}$ comparison is significant at the 5 per cent level in the "Groningen" populations on occasion 1, indicating a difference in the level of heterozygosity between those environments which contained fig medium and those which did not. Table 8 shows that those environments which contained fig media support a higher level of heterozygosity. This effect is transitory; on occasion 2 the only between environment item significant is the $C_{2} \times$ Loci item (at the 1 per cent level). This indicates a difference in heterozygosity between environments containing potato medium and those which do not which is not consistent for all loci. In the "Chateau Tahbilk" series of populations, $C_{3}$ is highly significant on sampling occasion 1, a consequence of the reduced genic heterozygosity in environments with two types of food medium compared with environments with only one type or with three types. Again this effect is transitory, not being found on occasion 2, but the $C_{1} \times$ loci item is significant at the 5 per cent level on both sampling occasions, indicating a difference between those environments which contain fig medium and those which do not, which is not consistent over all loci. Thus these data provide little evidence for a general and sustained effect of environmental heterogeneity on genic heterozygosity at the loci under observation; the effects observed were either not consistent over occasions or over all loci. 


\section{Discussion}

Despite the large amount of effort expended in the study of electrophoretically detectable polymorphisms there is as yet no consensus as to the extent to which they are influenced by selection. However, this is unsurprising in view of the large proportion of work concentrated upon surveys of the extent and distribution of electophoretic variation in natural populations. The lack of information on the ecology and dynamics of the surveyed populations has meant sets of data so derived are usually compatible with either extreme neutralist or selectionist viewpoints. Thus a more fruitful approach to the problem is provided by the laboratory investigation of electrophoretic polymorphisms.

Laboratory investigation of electrophoretic polymorphism are of two main types. Firstly, for individual, amenable loci, the in vitro properties, such as catalytic efficiency and thermostability, are determined. It may then be possible to relate these properties to the fitness of individual genotypes under selective conditions in the laboratory, or to the distribution of genotypes in the natural environment. This approach has met with some success in demonstrating that genotypic properties vary in ways which can be realistically related to environmental variation (de Jong and Scharloo, 1976; Hickey, 1977; van Delden, Boerema and Kamping, 1978; Kamping and van Delden, 1978). However, there has been less success in demonstrating that these polymorphisms are balanced in the natural environment.

In the long term, single locus studies will have to extend their scope to consider interactions between the locus under direct ovservation and associated loci (e.g., loci controlling expression), and between these loci and the rest of the genome and the environment. In the short term, it is necessary to broadly determine the limits of the response of genetic variation to natural selection. This need has led to the adoption of a second approach to the laboratory investigation of electrophoretic polymorphism which is to study the behaviour of several loci simultaneously in experimental populations. This approach, which is the one adopted in this study, has the additional advantage that it allows the investigation of general models of polymorphism maintenance, particularly those predicting associations between environmental heterogeneity and genic heterozygosity. The disadvantage of this approach is that it is not possible to ascribe selectively mediated changes at the loci under observation to selection acting directly on those loci. This disadvantage is outweighed, however, by the generality of the method, the observation of selection at a number of loci demonstrates that selection is of importance in effecting allele frequency changes over a large fraction of the genome.

The design of the experiment described here incorporates several advances over earlier studies. The main features of the study are:

(i) The only environmental variation included in the design was spatial, and the range of environments encompassed three levels of spatial heterogeneity. The more heterogeneous environments contained discrete niches.

(ii) In the main body of the experiment all environments were fully replicated, this facilitates comprehensive analysis of the data.

(iii) The experiment was further replicated over two recently collected populations, which provides information on the generality of the results. 
The outcome of this study was unexpected in some of its aspects. The results have re-affirmed that changes in allele frequency at electrophoretically detected loci may be mediated by selective forces, as it has been possible to demonstrate, for most loci under study, that systematic changes are found which can only have been caused by selection. However, rapid changes in allele frequency have occurred which are not shared by replicates, and, with the population size maintained in the experimental environments during the course of the study, these changes are unlikely to be due to random genetic drift. Nonetheless, random processes may play an important role in the initiation of such differences. The replicate divergence could come about through an accumulation of random environmental differences between replicates which initiate small genetic differences which are then magnified by selection. Alternatively, genetic differences between replicates for rare, but selectively important, alleles could have been created at the initiation of the populations. However, this seems unlikely to be the cause of much divergence as the rare alleles (either cryptic alleles, within an electrophoretic mobility class, at the loci under observation, or, alleles at a locus closely linked to the loci under observation) would have to be under intense selection to produce replicate differences. A more interesting possibility is that the loci under observation are part of a small segment of chromosome on which alleles at several loci are being selected in concert. Individual combinations of alleles will all be relatively rare, and the combination or combinations of alleles of selective significance may have been sampled initially or created de novo by recombination in one but not the other replicates. Another interesting possibility is that small genetic founder differences between replicates or small initial environmental differences between replicates placed the replicates at different positions on the fitness surface, this resulting in the replicates diverging to different selective peaks, or taking different routes to the same peak. Any of these mechanisms suggests a surprising subtlety in the response of genetic variability to natural selection.

In the systematic effects of selection demonstrated by the analyses of phenotype frequency (table 2) the effect of occasions was more common then the effects of experimental environments. The manipulated experimental environments produce some phenotypic differentiation, although there is no evidence for a positive relationship between environmental heterogeneity and genic heterozygosity, but the overall laboratory environment has had a greater effect. In the natural environment mortality can be caused by many different factors such as temperature, humidity, predation and competition; the laboratory environment, however, places a large emphasis on just one component of mortality, competition. The majority of mortality in the population cages is due to larval competition for resources and it is perhaps this which causes the strong selection obviously imposed by the laboratory environment. The effect of occasions is thus perhaps particularly prominent because the initial populations had only recently been collected. Indeed, it may be the particular vigour of the selective forces imposed by the laboratory environment which has resulted in substantial genomic rearrangement and thus replicate divergence initiated by rare recombinational events.

There is no general similarity between the responses of individual loci in the "Chateau Tahbilk" and "Groningen" populations. Those loci which 
respond to the manipulated experimental environments in one population do not do so in the other, and in no case is there evidence for a similar directional selection in the two populations. The difference in response may arise because there are cryptic genetic differences (one electromorph representing different alleles in the two populations), or differences in the background genotype or different linkage relationships in the two populations. It is not so easy to suggest why there is a difference in the overall amount of change, as demonstrated by the phenotype analyses (tables 4 and 5) and the analyses of allele frequency (tables 6 and 7) between the two populations, the "Chateau Tahbilk" derived population showing a greater amount of change than the "Groningen" derived population. This differences cannot be due to population size differences, or to differences in the amount of linkage disequilibrium created at the initiation of the experiment. Thus, the extent of the response in the two populations must reflect genomic differences between the initial two populations, and may be some measure of the difference between the environment of the natural population and that of the laboratory population.

Selection has been demonstrated to affect a number of the loci under observation, but its action may be transmitted to those loci in a number of ways. The effects may result from selection solely at the loci under observation (albeit mediated by the background genotype) from selection on the locus under observation as part of a group of associated linked loci, or from selection on a linked locus or loci in linkage disequilibrium with the locus under observation. In this latter case, as the initial population samples were fairly large, this linkage disequilibrium will have been sampled from the original populations. This raises the question of why linkage disequilibrium is present in the original population (a survey of the "Chateau Tahbilk" population shows that inversions are not a complication, at least in this population, those that were present at the end of this study were small and rare: Middleton, pers. comm.). Furthermore, in this case, the observed selection is a fair reflection of what might have happened in the natural population had it been exposed to a novel environment. Thus, even if the alleles under observation are themselves neutral, it may not be possible to describe their behaviour in the natural population by application of the neutral theory.

Acknowledgements. The Science and Engineering Research Council are thanked for a research studentship (C. S. Haley). Statistical calculations were carried out on the University of Birmingham ICL 1906A and DEC 2060 computers.

\section{REFERENCES}

AYALA, F. J. 1972. Darwinian versus non-Darwinian evolution in natural populations of Drosophila. Proc. Sixth Berkeley Symp. Math. Stat. Prob., Vol. V, 211-236.

BARKER, J. S. F. 1962. The estimation of generation interval in experimental populations of Drosophila. Genet. Res., 3, 388-404.

CROW, J. F. AND MORTON, N. E. 1955. Measurement of gene frequency drift in small populations. Evolution, 9, 202-214.

DELDEN, VAN W., BOEREMA, A. C. AND KAMPING, A. 1978. The alcohol dehydrogenase polymorphism in populations of Drosophila melanogaster. I. Selection in different environments. Genetics, 90, 161-191.

DEMPSTER, E. 1955. Maintenance of genetic heterogeneity. Cold Spring Harbour Symp. Quant. Biol., 20, 25-32. 
FISHER, R. A. AND FORD, E. B. 1947. The spread of a gene in natural conditions in a colony of the moth Panaxia dominula L. Heredity, 1, 143-174.

GILLESPIE, J. H. 1974. The role of environmental grain in the maintenance of genetic variation. Amer. Nat., 108, 831-836.

GILLESPIE, J. H. 1976. A general model to account for enzyme variation in natural populations. II. Characterisation of the fitness function. Amer. Nat., 110, 809-821.

GILLESPIE, J. H. AND LANGLEY, C. H. 1974. A general model to account for enzyme variation in natural populations. Genetics, 76, 837-848.

HALDANE, J. B. S. AND JAYAKAR, S. D. 1963. Polymorphism due to selection of varied direction. J. Genet., 58, 291-317.

HICKEY, D. A. 1977. Selection for amylase allozymes in Drosophila melanogaster. Evolution, $31,800-804$.

DE JONG, G. AND SCHARLOO, W., 1976. Environmental determination of selective significance or neutrality of amylase variants in Drosophila melanogaster. 2 . The relationship between ADH activity and adult mortality. Biochem. Genet., 16, 541-551.

KAMPING, A. AND DELDEN, VAN. W., 1978. The alcohol dehydrogenase polymorphism in populations of Drosophila melanogaster. 2. The relationship between ADH activity and adult mortality. Biochem. Genet., 16, 541-551.

KULLBACK, S. 1959. Information theory and statistics. Wiley, New York.

LEVENE. H. 1953. Genetic equilibrium when more than one ecological niche is available. Amer. Nat., 87, 331-333.

LEVINS, R. 1968. Evolution in changing environments. Princeton University Press, Princetown, New Jersey.

LEVINS, R. AND MACARTHUR, R. 1966. The maintenance of genetic polymorphism in a spatially heterogeneous environment: variations on a theme by Howard Levene. Amer. Nat., 100, 585-589.

MAYNARD SMITH, J. 1966. Sympatric speciation. Amer. Nat., 100, 637-650.

MCDONALD, J. F. AND AYALA, F. J. 1974. Genetic response to environmental heterogeneity. Nature, 250, 572-574.

MINAWA, A. AND BIRLEY, A. J. 1975. Genetical and environmental diversity in Drosophila melanogaster. Nature, 255, 702-704.

MINAWA, A. AND BIRLEY, A. J. 1978. The genetical response to natural selection by varied environments. I. Short-term observations. Heredity, 40, 39-50.

POULIK, M. D. 1957. Starch gel electrophoresis in a discontinuous system of buffers. Nature, $180,1477$.

POWELL, J. R. 1971. Genetic polymorphism in varied environments. Science, 174, 1035-1036. STROBECK, C. 1975. Selection in a fine grained environment. Amer. Nat., 109, 419-425.

TEMPLETON, A. R. AND ROTHMAN, E. D. 1974. Evolution in heterogeneous environments. Amer. Nat., 108, 409-428.

WALLIS, B. B. AND FOX, A. S. 1968. Genetic and developmental relationships between two alkaline phosphatases in Drosophila melanogaster. Biochem. Genet., 2, 141-158. 